\title{
EE-drospirenone-levomefolate calcium versus EE-drospirenone + folic acid: folate status during 24 weeks of treatment and over 20 weeks following treatment cessation
}

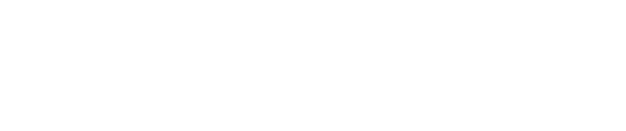

\author{
Konstanze Diefenbach' \\ Dietmar Trummer' \\ Frank Ebert ${ }^{\prime}$ \\ Michael Lissy² \\ Manuela Koch ${ }^{2}$ \\ Beate Rohde' \\ Hartmut Blode ${ }^{3}$ \\ 'Bayer HealthCare Pharmaceuticals, \\ Berlin, Germany; ${ }^{2}$ Nuvisan GmbH, \\ Neu-Ulm, Germany; ${ }^{3}$ Bayer \\ HealthCare Pharmaceuticals \\ Global R\&D Center, Beijing, \\ People's Republic of China
}

Correspondence: Hartmut Blode Bayer HealthCare Pharmaceuticals Global R\&D Center, I9F, Tower B, Bayer Center, 27 Dong San Huan North Road, Chaoyang District, Beijing 100020, People's Republic of China

Tel +86 I065360869

Email hartmut.blode@bayer.com
Background: Adequate folate supplementation in the periconceptional phase is recommended to reduce the risk of neural tube defects. Oral contraceptives may provide a reasonable delivery vehicle for folate supplementation before conception in women of childbearing potential. This study aimed to demonstrate that a fixed-dose combination of an oral contraceptive and levomefolate calcium leads to sustainable improvements in folate status compared with an oral contraceptive + folic acid.

Methods: This was a double-blind, randomized, parallel-group study in which 172 healthy women aged 18-40 years received ethinylestradiol (EE)-drospirenone-levomefolate calcium or EE-drospirenone + folic acid for 24 weeks (invasion phase), and EE-drospirenone for an additional 20 weeks (folate elimination phase). The main objective of the invasion phase was to examine the area under the folate concentration time-curve for plasma and red blood cell $(\mathrm{RBC})$ folate, while the main objective of the elimination phase was to determine the duration of time for which $\mathrm{RBC}$ folate concentration remained $\geq 906 \mathrm{nmol} / \mathrm{L}$ after cessation of EEdrospirenone-levomefolate calcium.

Results: Mean concentration-time curves for plasma folate, $\mathrm{RBC}$ folate, and homocysteine were comparable between treatment groups during both study phases. During the invasion phase, plasma and RBC folate concentrations increased and approached steady-state after about 8 weeks (plasma) or 24 weeks (RBC). After cessation of treatment with levomefolate calcium, folate concentrations decreased slowly. The median time to RBC folate concentrations falling below $906 \mathrm{nmol} / \mathrm{L}$ was 10 weeks (95\% confidence interval 8-12 weeks) after cessation of EE-drospirenone-levomefolate calcium treatment. Plasma and RBC folate levels remained above baseline values in $41.3 \%$ and $89.3 \%$ of women, respectively, at the end of the 20 -week elimination phase.

Conclusion: Improvements in folate status were comparable between EE-drospirenonelevomefolate calcium and EE-drospirenone + folic acid. Plasma and RBC folate levels remained elevated for several months following cessation of treatment with EE-drospirenone-levomefolate calcium.

Keywords: drospirenone, ethinylestradiol, folic acid, levomefolate calcium, neural tube defect, oral contraception

\section{Introduction}

Folate is the generic name for many different forms of this water-soluble vitamin, which include, among others, L-5-methyltetrahydrofolate (L-5-methyl-THF) and 
folic acid. Folates are essential for DNA and protein synthesis and, hence, cell division. Low folate levels are known to be linked to neural tube defects.

Neural tube defects are a complex group of congenital malformations that occur as a result of incomplete or incorrect closure of the neural tube (eg, anencephaly and spina bifida) during the early stages of pregnancy, often before a woman knows she is pregnant. ${ }^{1}$ While the etiology of neural tube defects remains poorly understood, the Medical Vitamin Research Council study published in $1991^{2}$ provided supportive evidence that an increased folate status is associated with reduced risk of neural tube defects, and in 1995 a quantitative relationship between reduction in risk of neural tube defects and red blood cell (RBC) folate levels was established by Daly et al. ${ }^{3}$ An RBC folate concentration $\geq 906 \mathrm{nmol} / \mathrm{L}$ was shown to be associated with a very low risk of neural tube defects $(0.8$ per 1000 births $){ }^{3}$

A number of initiatives have been undertaken to improve folate status among women of childbearing potential, ${ }^{4}$ including educational campaigns, food fortification programs, and policies to encourage folic acid supplementation during the periconceptional period (ie, both before and during the initial weeks of pregnancy). Current recommendations in the US and Europe specify that all women planning or capable of pregnancy should take folic acid supplements at doses of $0.400 \mathrm{mg} /$ day (0.400-0.800 mg/day in the US), commencing at least one month before conception. ${ }^{5,6}$ Indeed, there is now a large body of evidence to suggest that the occurrence or recurrence of neural tube defects can be dramatically reduced if women use folate supplements both before and during the initial weeks of pregnancy. ${ }^{2,7-17}$

However, initiatives to improve folate status among women of childbearing potential have, to date, had unsatisfying results. ${ }^{4,18,19}$ Four studies that assessed the effects of periconceptional folic acid usage before and after the introduction of public folic acid awareness campaigns in the UK, The Netherlands, and Australia showed post-campaign rates of folic acid use did not exceed $50 \% .{ }^{19}$ In the US, data from the National Health and Nutrition Examination Survey showed that only $24 \%$ of nonpregnant women of childbearing potential were consuming the recommended intake of folate. ${ }^{20}$ Thus, despite the knowledge that adequate folate status during the periconceptional period is associated with a reduced risk of neural tube defects, a gap remains in translating this knowledge into practical solutions that are effective in real-life situations, so additional efforts are needed. ${ }^{4,21}$

Oral contraceptives have been recommended as a reasonable delivery vehicle for folate supplementation in women of childbearing age. ${ }^{22}$ Two oral contraceptives, one containing ethinylestradiol (EE) $0.020 \mathrm{mg}$-drospirenone $3 \mathrm{mg}$-levomefolate calcium $0.451 \mathrm{mg}$ and one containing EE $0.030 \mathrm{mg}$-drospirenone $3 \mathrm{mg}$-levomefolate calcium $0.451 \mathrm{mg}$, were approved by the US Food and Drug Administration in 2010 for the improvement of folate status in women who desire contraception. ${ }^{23}$ Levomefolate calcium (Metafolin ${ }^{\circledR}$, Merck KGaA, Darmstadt, Germany), the calcium salt of L-5-methyl-THF (the predominant form of dietary folate and the principal form of circulating folate in the body), offers an alternative to folic acid for the improvement of folate status. Studies have shown that levomefolate calcium is at least as effective as folic acid at increasing RBC folate levels and reducing plasma homocysteine levels. ${ }^{24-27}$ Levomefolate calcium is considered to have advantages over folic acid. For example, levomefolate calcium is less likely to mask symptoms of vitamin $B_{12}$ deficiency, which is a concern with folic acid supplementation. ${ }^{28}$ In addition, there have been concerns about the presence of unmetabolized folic acid in the peripheral circulation; these would be alleviated with levomefolate calcium. ${ }^{28}$

This paper outlines findings from a long-term twophase study comparing the fixed-dose oral contraceptive formulation EE $0.030 \mathrm{mg}$-drospirenone $3 \mathrm{mg}$-levomefolate calcium $0.451 \mathrm{mg}$ with the free combination of oral contraceptive and equimolar folic acid with respect to folate status during 24 weeks of folate treatment and 20 weeks of follow-up without folate intake in healthy women of childbearing potential.

\section{Materials and methods Study design}

This was a randomized, double-blind, double-dummy (ie, throughout treatment, all women received both a tablet and a capsule, one of which was active and one of which was placebo, depending on the treatment group), parallel-group, single-center (Nuvisan GmbH, Neu-Ulm, Germany) study conducted between December 2006 and January 2008. The study was performed in accordance with the ethical principles stipulated by the Declaration of Helsinki and the International Conference on Harmonization/Good Clinical Practice. The study was approved by an independent ethics committee/institutional review board (Ethik-Komission der Bayerischen Landesärztekammer, München, Germany). All women provided written informed consent prior to study entry. The study was divided into two phases, ie, a 24 -week folate invasion phase followed by a 20 -week folate elimination phase. 


\section{Study participants}

Healthy women aged 18-40 years (inclusive) desiring contraception were eligible for inclusion. Participants were selected from the local population in accordance with inclusion and exclusion criteria. At screening, women were required to have plasma vitamin $B_{12}$ concentrations $\geq 110 \mathrm{pmol} / \mathrm{L}$ and $\mathrm{RBC}$ folate levels $>317 \mathrm{nmol} / \mathrm{L}$ and $<906 \mathrm{nmol} / \mathrm{L}$. Smokers were permitted, if they were aged between 18 and 30 years. Exclusion criteria included the usual contraindications for combined oral contraceptive use ${ }^{29}$ and: any disease or condition that could compromise the pharmacokinetics of the study medication; any disease or condition that could interfere with conduct of the study or interpretation of its results; intake within 4 weeks prior to study start of any drug known to induce or inhibit liver enzymes; current treatment with any medications that have the potential to interfere with folate metabolism (eg, methotrexate, trimethoprim, antacids, or antiepileptic drugs); or regular consumption of folic acid in vitamin supplements or pharmaceuticals during the preceding 4 months.

\section{Study treatments}

The treatment schedule is shown in Figure 1. For the 24-week (six-cycle) invasion phase, women were randomized in a 1:1 ratio to EE $0.030 \mathrm{mg}$-drospirenone $3 \mathrm{mg}$-levomefolate calcium $0.451 \mathrm{mg}$ (equivalent to L-5-methyl-THF $0.416 \mathrm{mg}$ ) or EE $0.030 \mathrm{mg}$-drospirenone $3 \mathrm{mg}+$ folic acid $0.400 \mathrm{mg}$ (equimolar to levomefolate calcium $0.451 \mathrm{mg}$ ).

Women in the levomefolate calcium group received a tablet containing EE $0.030 \mathrm{mg}$-drospirenone $3 \mathrm{mg}-$ levomefolate calcium $0.451 \mathrm{mg}$ and a placebo capsule once daily for 21 days, followed by a tablet containing levomefolate

\section{Invasion phase}

(6 cycles, 24 weeks)

\begin{tabular}{|c|c|}
\hline \multicolumn{2}{|c|}{ Levomefolate calcium group } \\
\hline EE-drsp-levomefolate calcium tablets & Levomefolate calcium tablets \\
\hline AND & AND \\
\hline Placebo capsules & Placebo capsules \\
\hline For 21 days & $\begin{array}{l}\left(\mathrm{HFI}^{\mathrm{b}}\right) \\
\text { For } 7 \text { days }\end{array}$ \\
\hline $\mathrm{L}$ & 1 \\
\hline Day 1 & Day 21 \\
\hline
\end{tabular}

\begin{tabular}{|c|c|}
\hline \multicolumn{2}{|c|}{ Folic acid group } \\
\hline EE-drsp tablets ${ }^{\circ}$ & Placebo tablets \\
\hline AND & AND \\
\hline Folic acid capsules & Folic acid capsules \\
\hline For 21 days & $\begin{array}{l}\left(\mathrm{HFI}^{\mathrm{e}}\right) \\
\text { For } 7 \text { days }\end{array}$ \\
\hline L & 1 \\
\hline Day 1 & Day 21 \\
\hline
\end{tabular}

\section{Elimination phase}

(5 cycles, 20 weeks)

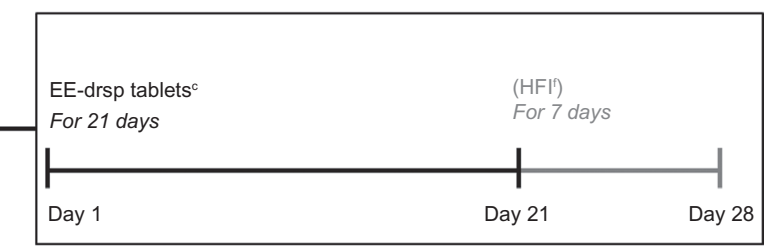

Figure I Treatment schedule.

Notes: Cycles were repeated five times (ie, six treatment cycles) during the 24-week invasion phase and four times (ie, five treatment cycles) during the 20-week elimination phase. All treatments were administered once daily. ${ }^{\mathrm{a} E E} 0.030 \mathrm{mg}$-drospirenone $3 \mathrm{mg}$-levomefolate calcium $0.45 \mathrm{I} \mathrm{mg}$ tablet; blevomefolate calcium $0.45 \mathrm{I}$ mg tablet AND

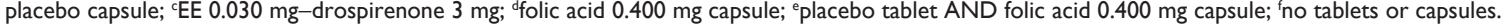
Abbreviations: drsp, drospirenone; $\mathrm{EE}$, ethinylestradiol; HFI, hormone-free interval. 
calcium $0.451 \mathrm{mg}$ only and a placebo capsule for 7 days. In the folic acid group, women took a tablet containing EE $0.030 \mathrm{mg}$-drospirenone $3 \mathrm{mg}$ and a capsule containing folic acid $0.400 \mathrm{mg}$ once daily for 21 days, followed by a placebo tablet and a folic acid capsule once daily for 7 days. These cyclic regimens were administered six times, ie, for 24 weeks. To maintain blinding, hormone tablets (with or without levomefolate calcium) and placebo tablets were identical in appearance, as were folic acid capsules and placebo capsules.

In the subsequent elimination phase, both groups received EE $0.030 \mathrm{mg}$ - drospirenone $3 \mathrm{mg}$ for a further five cycles (20 weeks). During the elimination phase, each cycle consisted of once-daily hormone treatment for 21 consecutive days, followed by a tablet-free interval of 7 days.

All study medication was provided in identical blister packs to maintain blinding. During the course of the study, women were not allowed to take vitamin supplements or any medications that contained folate or interacted with folate absorption, metabolism, or excretion.

\section{Study assessments}

For the determination of plasma and RBC folate, homocysteine and plasma folate metabolites, fasting venous blood samples were collected in plasma tubes coated with lithiumheparin. Samples were collected by trained staff and analyzed at a central laboratory.

\section{Baseline levels}

Plasma folate, RBC folate, and plasma homocysteine levels at baseline (ie, at the start of the invasion phase) were determined on three separate occasions (minimum of 5 days apart including one weekend) before any study medication was received. The median of the pretreatment levels was taken to represent the respective individual baseline values.

\section{Plasma and RBC folate levels}

Samples were collected at baseline and at biweekly intervals after the start of treatment and were used to determine plasma and whole blood folate levels. Aliquots of whole blood were lyzed with freshly prepared 1\% ascorbic acid (1:10 dilution), vortexed, and incubated for 30 minutes in the dark before storage at approximately $-80^{\circ} \mathrm{C}$ until analysis. The remaining whole blood sample was centrifuged to obtain plasma, which was separated and stored frozen at approximately $-80^{\circ} \mathrm{C}$ until analysis.

Plasma and whole blood folate concentrations were determined using a validated microbiologic assay at Toegepast Natuurwetenschappelijk Onderzoek (Netherlands
Organisation for Applied Scientific Research), Zeist, The Netherlands. Folate concentrations were calculated using an appropriate calibration curve, with folic acid as a reference (range $25-500 \mathrm{ng} / \mathrm{mL}$ for whole blood folate and 1-20 ng/mL for plasma folate). Each plate also included a World Health Organization folate standard control.

Based on the results of quality control sample analyses, precision was deemed to be $5 \%-18 \%$ in plasma and $8 \%-9 \%$ in whole blood, and accuracy was $97 \%-107 \%$ in plasma and $100 \%-104 \%$ in whole blood. RBC folate concentrations were calculated using the following equation (where hematocrit is given as a percentage of whole blood volume):

$$
\text { RBC folate }=\frac{-[\text { Plasma folate } *(100-\text { Hematocrit })]}{\text { Hematocrit }}
$$

For RBC folate, validation rules were put in place to control for unusually high whole blood folate levels (which were deemed to be due to incorrect dilution). Samples with either folate concentrations $>120 \%$ of the upper limit of the calibration curve or hematocrit values outside the usual intraindividual variability were deemed invalid.

\section{Plasma homocysteine}

Samples were collected at baseline and then every 2 weeks. Plasma was separated by centrifugation at $4^{\circ} \mathrm{C}$ and stored frozen at approximately $-20^{\circ} \mathrm{C}$ until analysis. Homocysteine concentrations were determined by fluorescence polarization immunoassay using the Abbott AxSym analyzer, (Abbott Diagnostics, Lake Forest, IL, USA) a method that shows good accuracy and precision. ${ }^{30}$

\section{Plasma folate metabolites}

The main circulating folate metabolites [folic acid, tetrahydrofolate (THF), 5-methyl-THF, 5-formyl-THF/10-formylTHF, and 5,10-methenyl-THF] were determined in plasma prior to treatment (baseline) and at week 12 and week 24 of treatment. The dihydrofolate metabolite was not analyzed because of its known instability and conversion to folic acid. In addition, no discrimination between 5-formyl-THF and 10-formyl-THF was possible due to the known interconversion of 10-formyl-THF to 5-formyl-THF. ${ }^{31}$

Plasma was obtained by centrifugation and stored frozen at approximately $-80^{\circ} \mathrm{C}$ before analysis. Plasma folate metabolites were determined using a validated liquid chromatography-mass spectrometry method, which was characterized as semiquantitative because the acceptance criteria for precision and accuracy were set at $50 \%$ (based on quality 
control sample analyses). The lower level of quantification was as follows for each of the metabolites: folic acid, 3.398-3.710 nmol/L; L-5-methyl-THF, 3.325-3.680 nmol/L; 5-formyl-THF/10-formyl-THF, 1.105-1.260 nmol/L; THF, 3.447-3.539 nmol/L; and 5,10-methenyl-THF, $1.052-1.284 \mathrm{nmol} / \mathrm{L}$.

\section{Compliance}

Compliance was assessed from participants' recordings of tablet intake in diaries along with the return of all unused study medication and empty blister packs at each visit.

\section{Nutritional intake}

To evaluate dietary folate intake, subjects completed a standardized food questionnaire provided by the University of Bonn. The prospective questionnaire was completed for 3 days at admission and at the end of weeks 8, 16, and 24 .

\section{Safety}

Safety was assessed in the full analysis set, which comprised all women who were treated with at least one dose of the study drug, and for whom at least one clinical observation after the start of treatment was available. Evaluations included physical and gynecologic examinations, assessment of vital signs, cervical smear tests, laboratory testing, and monitoring of adverse events. Adverse events that occurred during the study were coded using the Medical Dictionary for Regulatory Activities and assessed by intensity, seriousness, pattern, and relationship to study medication or study conduct (related [possible, probable, and definite]/not related [none, unlikely]). Safety outcomes are presented separately for the invasion phase and elimination phase.

\section{Statistical analyses and power calculations}

The objectives were different for the invasion and elimination phases of the study.

\section{Invasion phase}

The primary objective of the invasion phase of this study was to examine whether EE-drospirenone-levomefolate calcium and EE-drospirenone + folic acid produce similar plasma and similar RBC folate exposures (as measured by the area under the folate concentration-time curve over 24 weeks $\left[\mathrm{AUC}_{(0-24 \text { weeks })}\right]$ for plasma and RBC folate). These values were chosen as primary variables because, unlike selected individual time points, they integrate changes in these variables over the complete folate treatment period and, thus, provide a more stable parameter for comparison between study groups.
The means of $\mathrm{AUC}_{(0-24 \text { weeks })}$ values on the log scale were estimated using an analysis of variance model with treatment as a factor. Two-sided 90\% confidence intervals (CIs) for the test (EE-drospirenone-levomefolate calcium) to reference (EE-drospirenone + folic acid) geometric mean ratio of the $\mathrm{AUC}_{(0-24 \text { weeks) }}$ were calculated for plasma and $\mathrm{RBC}$ folate exposure to assess whether they were contained within the limits for bioequivalence (range 80\%-125\%). Plasma and RBC folate $\mathrm{AUC}_{(0-24 \text { weeks })}$ values, uncorrected or corrected for baseline folate concentrations, were analyzed with inferential statistical methods using the per protocol set, which consisted of all treated women who did not present any major protocol deviations.

Other variables assessed during the invasion phase included change in homocysteine levels between baseline and 24 weeks, and plasma folate metabolite patterns at baseline and at 12 and 24 weeks. All secondary variables were analyzed descriptively. Absolute values and change from baseline in plasma folate, $\mathrm{RBC}$ folate, and homocysteine levels were also assessed using descriptive statistics.

\section{Elimination phase}

The main objective of the elimination phase of this study was to determine the duration of time for which RBC folate concentration remained $\geq 906 \mathrm{nmol} / \mathrm{L}$ after cessation of folate treatment in women who received EEdrospirenone-levomefolate calcium during the 24-week invasion phase. The duration that $\mathrm{RBC}$ folate concentrations were $\geq 906 \mathrm{nmol} / \mathrm{L}$ was calculated using the Kaplan-Meier estimator including the two-sided $95 \%$ CI for the median time taken for RBC folate concentration to fall below $906 \mathrm{nmol} / \mathrm{L}$. These analyses were undertaken using the per protocol set.

Secondary variables in the elimination phase were assessed in both treatment groups using descriptive statistics and included: mean concentrations, mean changes from baseline, and mean changes from the end of the folate invasion phase (week 24) for plasma folate, RBC folate, and homocysteine at biweekly time points during the elimination phase (weeks 26-44), the proportion of women with $\mathrm{RBC}$ and plasma folate levels below their median baseline (prefolate treatment) level, and the proportion of women with homocysteine levels above their median baseline (prefolate treatment) level at biweekly time points from weeks 26-44.

\section{Sample size}

Taking into account the special design as a long-term bioequivalence study, scheduling a high number of visits with 
blood testing over 24 weeks (the period on which the sample size calculation was based), it was expected that the dropout rate in this study would be higher than that observed in other contraceptive studies. Assuming that $37 \%$ of participants would not be included in the per protocol set and that the true ratio of test-to-reference $\mathrm{AUC}_{(0-24 \text { weeks })}$ was $110 \%$, then enrolment of 86 women per treatment group (172 women overall) was required to achieve bioequivalence (limits of $80 \%-125 \%$ ) with $90 \%$ power. Based on additional analyses of previously published data, ${ }^{24}$ a precision of 0.24 standard deviations of difference on the log-scale was assumed. Although the determination of sample size was based on plasma folate, no adjustment of sample size after inclusion of RBC folate as the primary endpoint was considered necessary because both primary endpoints were expected to be highly correlated.

\section{Results}

Overall, 235 women were screened, of whom 172 (EEdrospirenone-levomefolate calcium, $\mathrm{n}=86$; EE-drospirenone + folic acid, $n=86$ ) were randomized to treatment. Of the 63 women who were excluded, 46 did not meet the inclusion criteria (three due to low vitamin $\mathrm{B}_{12}$ levels; none of the women were excluded due to low RBC folate levels), eight withdrew consent, eight were excluded for logistical reasons (ie, the study dates conflicted with planned holidays; these patients were classified as screening failures), and one was excluded because blood sampling was not possible. Women who received study medication and had at least one clinical observation after the start of treatment were included in the full analysis set. Over 44 weeks (both the invasion and elimination phases), 12 women prematurely discontinued the study medication (Figure 2). Major protocol deviations

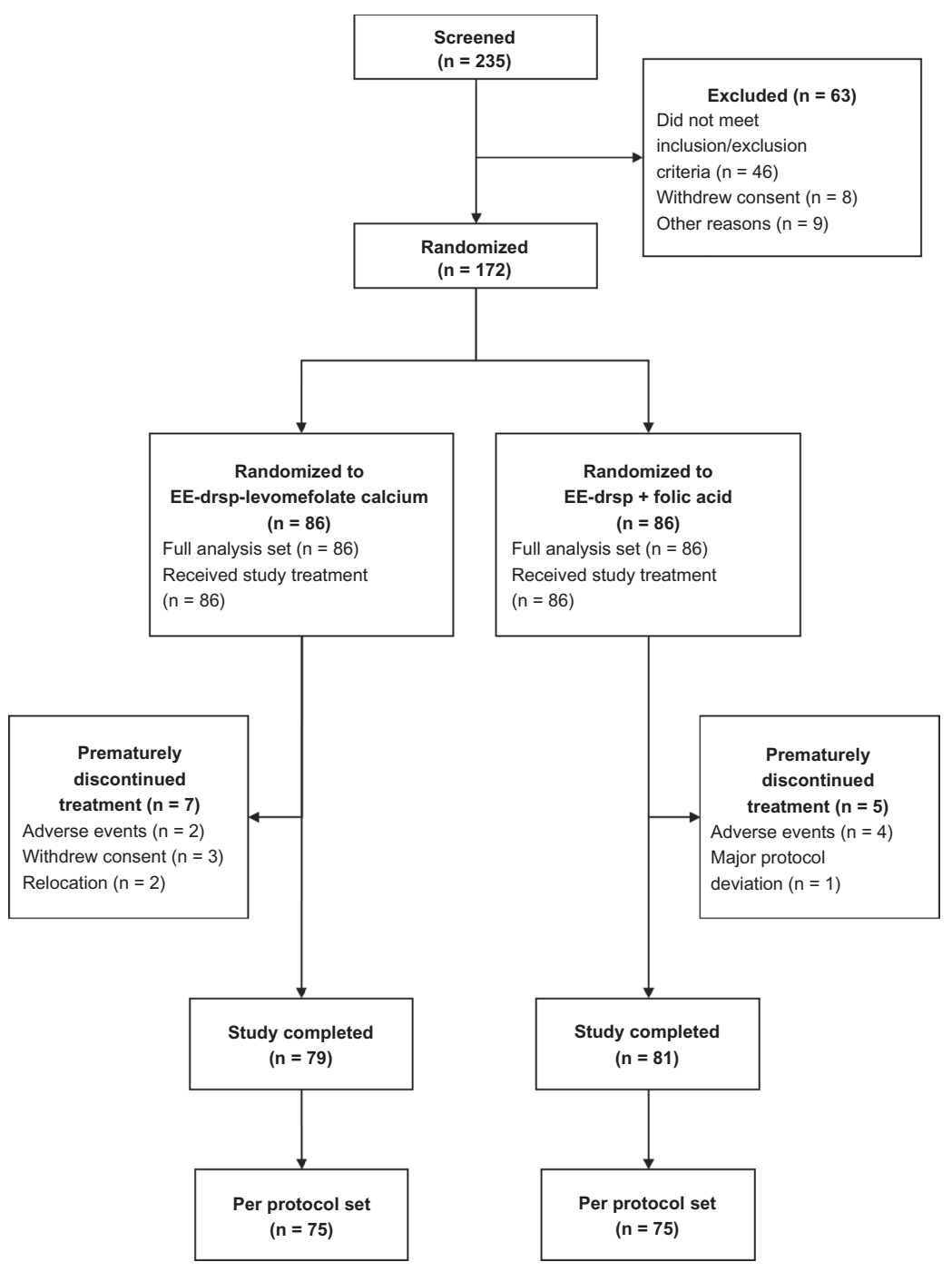

Figure 2 Disposition of women throughout the study. Abbreviations: drsp, drospirenone; $\mathrm{EE}$, ethinylestradiol. 
(all of which were due to missing valid data) were noted in 11 women in each treatment group. Analysis of the primary variables was performed in 150 women (EE-drospirenonelevomefolate calcium, $\mathrm{n}=75$; EE-drospirenone + folic acid, $n=75$ ) who showed no major protocol deviations and comprised the per protocol set.

All study participants were Caucasian with a mean age of 27.7 (range 18-40) years. Baseline and demographic characteristics for the per protocol set are reported in Table 1 and were comparable with those in the full analysis set (data not shown). Compliance was high in both treatment groups over 44 weeks ( 11 cycles); the range of means was 27.6-28.1 tablets/capsules per 28-day cycle. Nutrition assessments were available for 150 women. Mean total dietary folate intake was not substantially different at the four assessment time points (range $0.176 \pm 0.070$ to $0.209 \pm 0.196 \mathrm{mg} /$ day).

\section{Plasma and RBC folate exposure during the invasion phase}

The geometric mean $\mathrm{AUC}_{(0-24 \text { weeks })}$ for plasma and $\mathrm{RBC}$ folate were generally comparable following treatment with EE-drospirenone-levomefolate calcium and EEdrospirenone + folic acid (baseline-uncorrected and baseline-corrected values, Table 2). In both treatment groups, variability was higher for baseline-corrected values of $\mathrm{AUC}_{(0-24 \text { weeks })}$ for plasma and $\mathrm{RBC}$ folate. The geometric mean ratios (EE-drospirenone-levomefolate calcium versus EE-drospirenone + folic acid) for baselineuncorrected and baseline-corrected plasma $\mathrm{AUC}_{(0-24 \text { weeks }}$ were 114\% (two-sided 90\% CI 107\%-121\%) and 114\% (two-sided 90\% CI 105\%-124\%), respectively. Exposure was similar between treatments using bioequivalence criteria (defined as two-sided $90 \%$ CI limits of $80 \%-125 \%$ ); corresponding geometric mean ratios for baseline-uncorrected and baseline-corrected $\mathrm{RBC}$ folate $\mathrm{AUC}_{(0-24 \text { weeks })}$

Table I Baseline and demographic characteristics of study participants (per protocol set)

\begin{tabular}{lll}
\hline & $\begin{array}{l}\text { EE-drsp-levomefolate } \\
\text { calcium } \\
(\mathbf{n}=\mathbf{7 5})\end{array}$ & $\begin{array}{l}\text { EE-drsp + } \\
\text { folic acid } \\
(\mathbf{n}=\mathbf{7 5})\end{array}$ \\
\hline Age, years & $28.2 \pm 5.7$ & $27.0 \pm 5.5$ \\
Height, $\mathrm{cm}$ & $165.9 \pm 6.3$ & $167.1 \pm 5.5$ \\
Body weight, $\mathrm{kg}$ & $63.3 \pm 8.2$ & $62.8 \pm 8.6$ \\
Body mass index, $\mathrm{kg} / \mathrm{m}^{2}$ & $23.0 \pm 2.6$ & $22.5 \pm 2.6$ \\
Current smoker, $\mathrm{n}(\%)$ & $22(29.3)$ & $21(28.0)$ \\
\hline
\end{tabular}

Note: Data are presented as the mean \pm standard deviation unless otherwise stated.

Abbreviations: drsp, drospirenone; EE, ethinylestradiol.
Table 2 Area under the folate concentration-time curve from time 0 (baseline) to week 24 ( $\mathrm{AUC}_{(0-24 \text { weeks) }}$ ) for plasma and RBC folate (uncorrected and corrected for baseline folate concentrations) after administration of EE-drospirenone-levomefolate calcium or EE-drospirenone + folic acid for 24 weeks (invasion phase; per protocol set)

\begin{tabular}{|c|c|c|c|c|}
\hline & \multicolumn{2}{|c|}{$\begin{array}{l}\text { Plasma folate } \\
\text { AUC }_{(0-24 \text { weeks) }} \\
\text { (nmol week/L) }\end{array}$} & \multicolumn{2}{|c|}{$\begin{array}{l}\text { RBC folate } A U C_{(0-24 \text { weeks })} \\
\text { (nmol week/L) }\end{array}$} \\
\hline & $\begin{array}{l}\text { Baseline- } \\
\text { uncorrected }\end{array}$ & $\begin{array}{l}\text { Baseline- } \\
\text { corrected }\end{array}$ & $\begin{array}{l}\text { Baseline- } \\
\text { uncorrected }\end{array}$ & $\begin{array}{l}\text { Baseline- } \\
\text { corrected }\end{array}$ \\
\hline $\begin{array}{l}\text { EE-drsp- } \\
\text { levomefolate } \\
\text { calcium } \\
(\mathrm{n}=75)\end{array}$ & $1030(24.0)$ & $640(29.0)$ & $24,176(21.8)$ & $\begin{array}{l}10,427 \\
(34.3)\end{array}$ \\
\hline $\begin{array}{l}\text { EE-drsp }+ \\
\text { folic acid } \\
(n=75)\end{array}$ & $904(22.3)$ & $56 \mid(32.7)$ & 21,876 (17.7) & $\begin{array}{l}8863 \\
(24.6)\end{array}$ \\
\hline
\end{tabular}

Note: Data presented as geometric mean (\% geometric coefficient of variation). Abbreviation: AUC, area under the concentration-time curve; drsp, drospirenone; $\mathrm{EE}$, ethinylestradiol; RBC, red blood cell.

were 111\% (two-sided 90\% CI 105\%-117\%) and $118 \%$ (two-sided 90\% CI 109\%-127\%), respectively.

\section{Plasma and RBC folate concentrations Invasion phase}

The mean concentration-time curves for plasma folate and $\mathrm{RBC}$ folate during the invasion phase were similar between EE-drospirenone-levomefolate calcium and EEdrospirenone + folic acid groups; however, the concentrations of both plasma and RBC folate were slightly higher with EE-drospirenone-levomefolate calcium (Figures 3 and 4).

Plasma folate levels increased from baseline, approaching a plateau after about 8 weeks in both treatment groups. Mean plasma folate concentrations increased in a cyclic pattern, and the highest plasma folate concentrations were reached at week 22 for both treatments (Figure 3). At week 24 of treatment, mean values were $49.9 \pm 15.5 \mathrm{nmol} / \mathrm{L}$ for $\mathrm{EE}-$ drospirenone-levomefolate calcium and $43.3 \pm 13.3 \mathrm{nmol} / \mathrm{L}$ for EE-drospirenone + folic acid.

For both treatments, RBC folate concentrations increased (Figure 4), reaching the highest mean concentrations (1361 $\pm 322 \mathrm{nmol} / \mathrm{L}$ for EE-drospirenone-levomefolate calcium and $1207 \pm 217 \mathrm{nmol} / \mathrm{L}$ for EE-drospirenone + folic acid) at week 24 . After 24 weeks of treatment, $95 \%$ of women had RBC levels $\geq 906 \mathrm{nmol} / \mathrm{L}$, the level associated with a very low risk of neural tube defects. ${ }^{3}$ Mean RBC folate levels of $\geq 906 \mathrm{nmol} / \mathrm{L}$ were reached after around 8 weeks of treatment with EE-drospirenone-levomefolate calcium. 


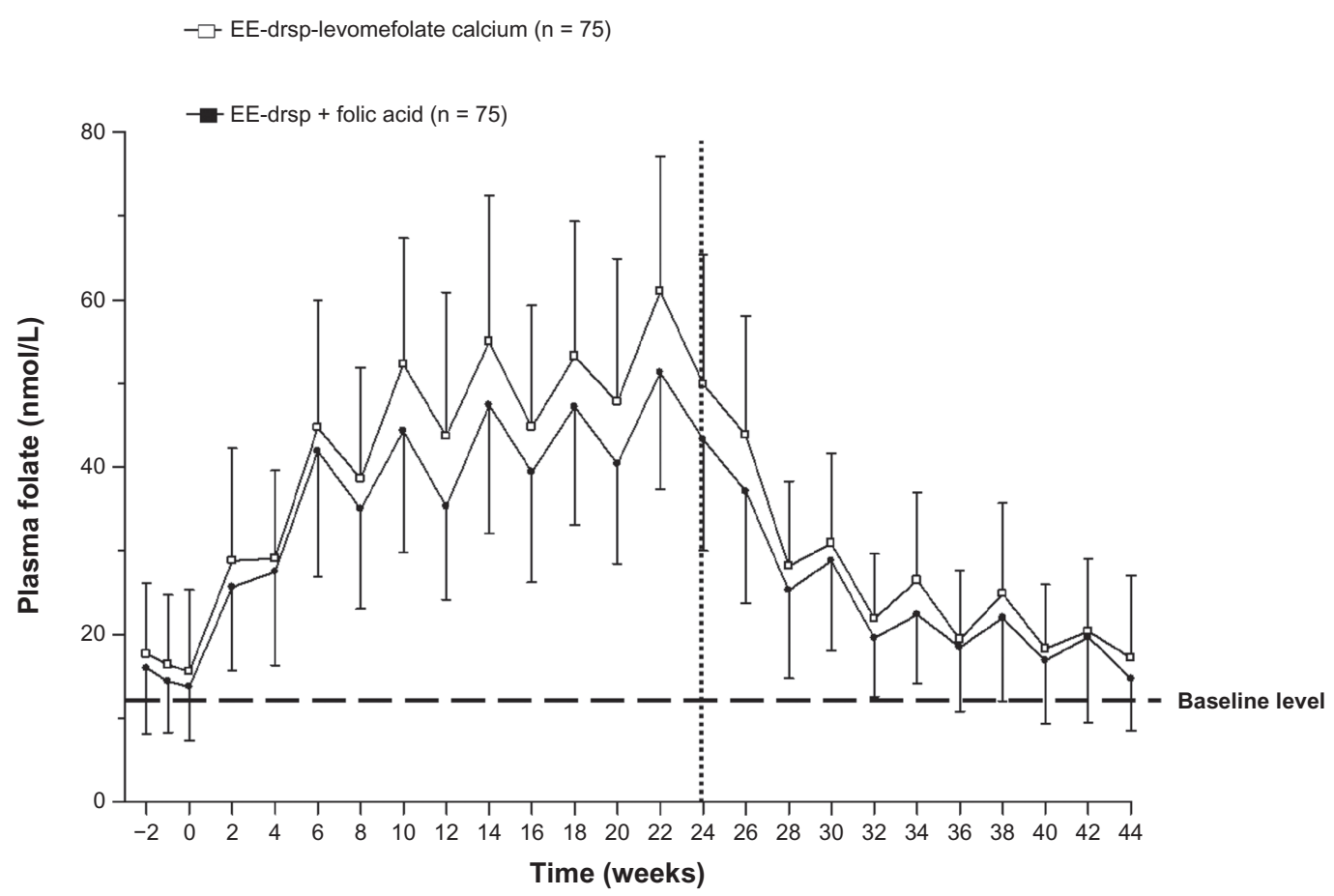

Figure 3 Concentration-time curves for plasma folate during 24 weeks of treatment with EE-drospirenone-levomefolate calcium or EE-drospirenone + folic acid (invasion phase) and during the 20-week period following cessation of treatment (elimination phase; per protocol set).

Notes: Vertical lines indicate the mean \pm standard deviation. Values prior to week 0 represent the three pretreatment measurements that were used to calculate the mean median baseline value. Vertical dotted line indicates the cutoff between the two phases.

Abbreviations: drsp, drospirenone; EE, ethinylestradiol.

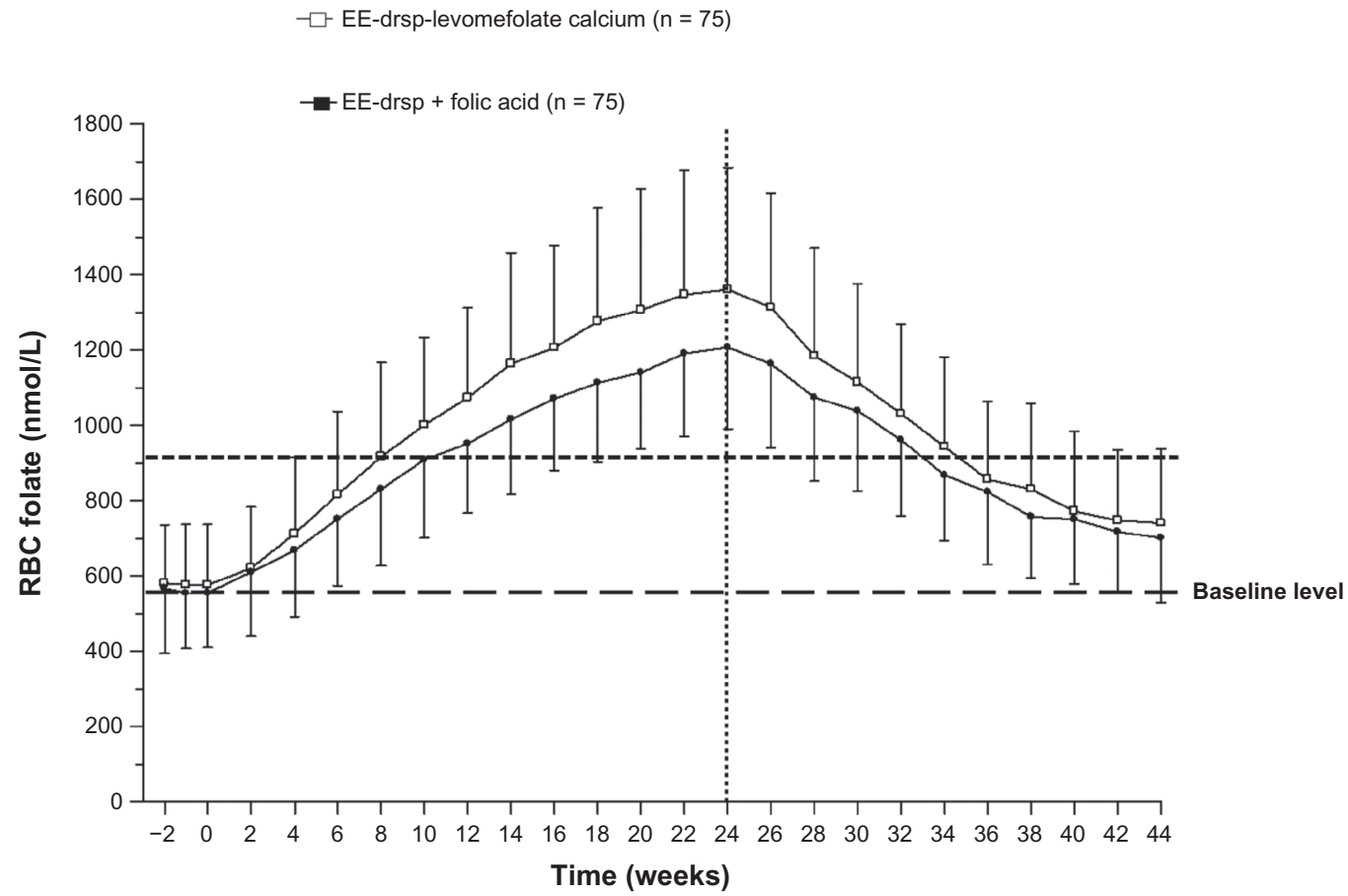

Figure 4 Concentration-time curves for RBC folate during 24 weeks of treatment with EE-drospirenone-levomefolate calcium or EE-drospirenone + folic acid (invasion phase) and during the 20 -week period following cessation of treatment (elimination phase; per protocol set).

Notes: Vertical lines indicate the mean \pm standard deviation. Values prior to week 0 represent the three pre-treatment measurements that were used to calculate the mean median baseline value. Vertical dotted line indicates the cutoff between the two phases.

Abbreviations: drsp, drospirenone; EE, ethinylestradiol; RBC, red blood cell. 


\section{Elimination phase}

The mean concentration-time curves for plasma and RBC folate during the elimination phase were comparable between groups (Figures 3 and 4). Plasma folate levels decreased at similar rates in both groups during the elimination phase, with approximately $40 \%$ of women in both groups maintaining plasma folate levels above those at baseline 20 weeks after the cessation of folate treatment (Table 3). Mean plasma folate values were $17.2 \pm 9.8 \mathrm{nmol} / \mathrm{L}$ on day 28 of the fifth treatment cycle after cessation of treatment (ie, week 44) in the EE-drospirenone-levomefolate calcium group. For the EE-drospirenone + folic acid group, mean plasma folate concentrations were $14.7 \pm 6.2 \mathrm{nmol} / \mathrm{L}$ at day 28 of cycle 5 .

The median time to RBC folate concentrations falling below $906 \mathrm{nmol} / \mathrm{L}$ was 10 weeks ( $95 \%$ CI $8-12$ weeks) after cessation of EE-drospirenone-levomefolate calcium treatment (ie, week 34 of the study; Figure 5). Although RBC folate levels decreased during the elimination phase in both groups of women, these levels remained above baseline for the majority (approximately $90 \%$ of women) at 20 weeks following cessation of folate treatment (Table 3 ). Four weeks after stopping folate intake, $85 \%$ of women maintained an $\mathrm{RBC}$ folate level above $906 \mathrm{nmol} / \mathrm{L}$, the level associated with very low risk of neural tube defects. ${ }^{3}$ At $8,10,12$, and 20 weeks after cessation of folate intake, the proportions of women with an RBC folate level above $906 \mathrm{nmol} / \mathrm{L}$ were $60 \%, 47 \%, 29 \%$, and $9 \%$, respectively. Mean RBC folate values at week 44 , ie, 20 weeks after cessation of treatment, were $739.8 \pm 197.6 \mathrm{nmol} / \mathrm{L}$ and $701.1 \pm 170.6 \mathrm{nmol} / \mathrm{L}$ for the EE-drospirenone-levomefolate calcium and EEdrospirenone + folic acid groups, respectively.

\section{Homocysteine levels}

Invasion phase

Homocysteine values decreased from baseline (Table 3), reaching a plateau after 8 weeks in both treatment groups and remained below baseline values during the entire folate administration period of 24 weeks. At the end of the invasion phase (week 24), the mean homocysteine values were $7.5 \pm 1.6 \mu \mathrm{mol} / \mathrm{L}$ and $7.6 \pm 1.4 \mu \mathrm{mol} / \mathrm{L}$, respectively, for EEdrospirenone-levomefolate calcium and EE-drospirenone + folic acid.

\section{Elimination phase}

Homocysteine levels began to increase following cessation of folate treatment. By week 44 of the elimination phase, homocysteine levels remained below baseline in $34.7 \%$ of women in the EE-drospirenone-levomefolate calcium group and $40.0 \%$ of women in the EE-drospirenone + folic acid group (Table 3). Mean homocysteine levels at week 44 were $9.0 \pm 2.6$ for EE-drospirenone-levomefolate calcium and $8.9 \pm 1.9$ for EE-drospirenone + folic acid.

\section{Folate metabolite pattern}

While plasma L-5-methyl-THF could be determined in all samples analyzed, most of the concentrations for the other plasma folate metabolites were found to be below the lower level of quantification. As for total folate, the concentrations of L-5-methyl-THF in plasma were comparable between both treatment groups, with an almost completely overlapping range of values (Figure 6). For 5,10-methenyl-THF and THF, concentrations above the

Table 3 Proportion of women (cumulative) with plasma and RBC folate levels below the mean median baseline value, and with homocysteine levels above the mean median baseline value in the 20 -week period following the cessation of 24 weeks of treatment with EE-drospirenone-levomefolate calcium or EE-drospirenone + folic acid (per protocol set)

\begin{tabular}{|c|c|c|c|c|c|c|}
\hline \multirow[t]{2}{*}{ Week } & \multicolumn{2}{|l|}{ Plasma folate } & \multicolumn{2}{|l|}{ RBC folate } & \multicolumn{2}{|l|}{ Homocysteine } \\
\hline & Levomefolate calcium & Folic acid & Levomefolate calcium & Folic acid & Levomefolate calcium & Folic acid \\
\hline 26 & $0(0.0)$ & $0(0.0)$ & $0(0.0)$ & $0(0.0)$ & $4(5.3)$ & $2(2.7)$ \\
\hline 28 & $4(5.3)$ & $4(5.3)$ & $0(0.0)$ & $0(0.0)$ & $8(10.7)$ & $5(6.7)$ \\
\hline 30 & $5(6.7)$ & $8(10.7)$ & $0(0.0)$ & $0(0.0)$ & II (14.7) & $9(12.0)$ \\
\hline 32 & $11(14.7)$ & $12(16.0)$ & $0(0.0)$ & $0(0.0)$ & $14(18.7)$ & $12(16.0)$ \\
\hline 34 & $15(20.0)$ & $17(22.7)$ & $\mathrm{I}(\mathrm{I} .3)$ & $0(0.0)$ & $16(21.3)$ & 14 (18.7) \\
\hline 36 & $23(30.7)$ & $23(30.7)$ & $2(2.7)$ & $2(2.7)$ & $19(25.3)$ & $17(22.7)$ \\
\hline 38 & $25(33.3)$ & $25(33.3)$ & $2(2.7)$ & $2(2.7)$ & $20(26.7)$ & $23(30.7)$ \\
\hline 40 & $30(40.0)$ & $34(45.3)$ & $6(8.0)$ & $2(2.7)$ & $22(29.3)$ & $26(34.7)$ \\
\hline 42 & $30(40.0)$ & $38(50.7)$ & $7(9.3)$ & $3(4.0)$ & $25(33.3)$ & $29(38.7)$ \\
\hline 44 & $44(58.7)$ & $45(60.0)$ & $8(10.7)$ & $6(8.0)$ & $26(34.7)$ & $30(40.0)$ \\
\hline
\end{tabular}

Notes: Data are $\mathrm{n}(\%)$. The mean median baseline values were as follows for EE-drospirenone-levomefolate calcium and EE-drospirenone + folic acid, respectively: plasma folate, 16.4 and I4.I nmol/L; RBC folate, 578.3 and $550.2 \mathrm{nmol} / \mathrm{L}$; homocysteine, 9.3 and $9.2 \mu \mathrm{mol} / \mathrm{L}$.

Abbreviations: EE, ethinylestradiol; RBC, red blood cells. 


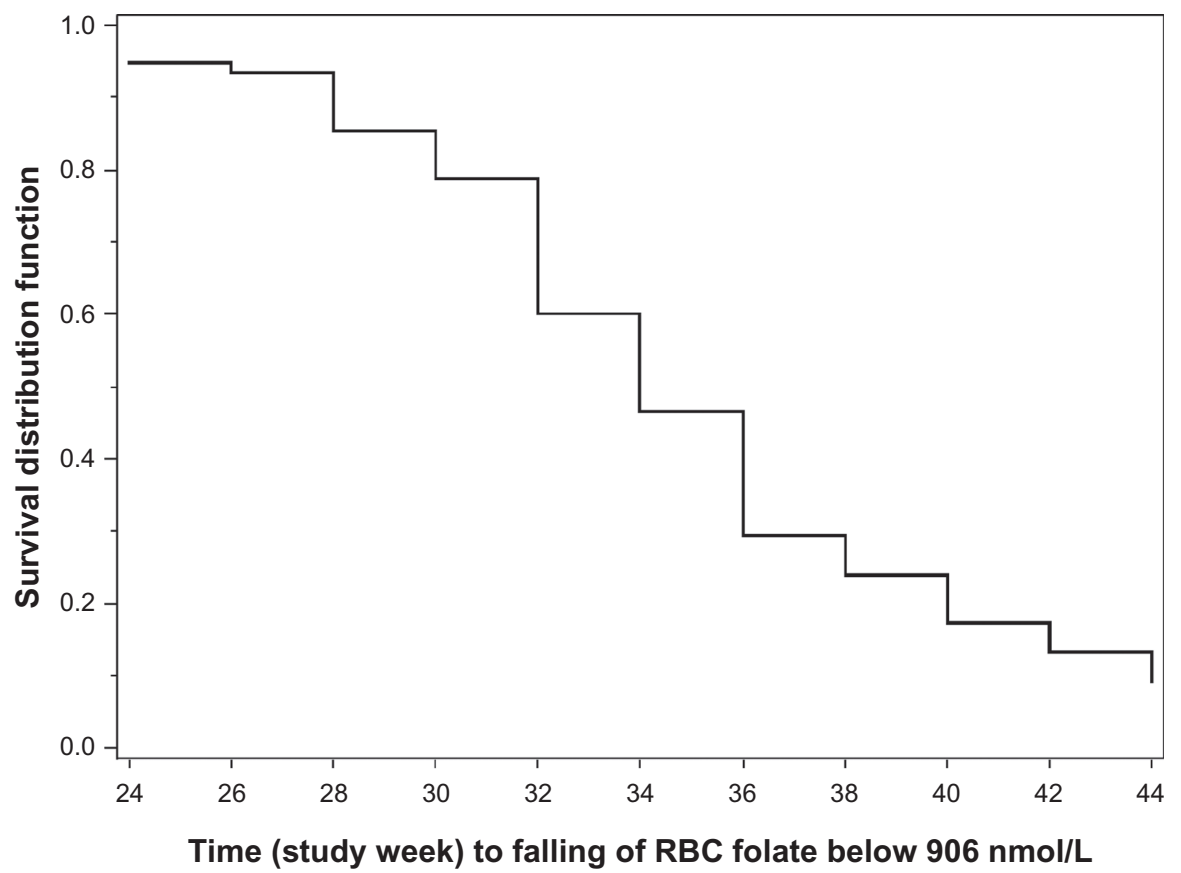

Figure 5 Kaplan-Meier estimates for time to RBC folate levels falling below $906 \mathrm{nmol} / \mathrm{L}$ for EE-drospirenone following treatment with EE-drospirenone-levomefolate calcium (per protocol set).

Abbreviations: EE, ethinylestradiol; RBC, red blood cell.

lower level of quantification were noted in plasma in some women (Table 4). Comparison of treatment groups did not reveal differences with regard to either the metabolite concentrations above the lower level of quantification or the percentage of women with metabolite concentrations below the lower level of quantification (Figure 7) following daily intake of equimolar doses of levomefolate calcium or folic acid.

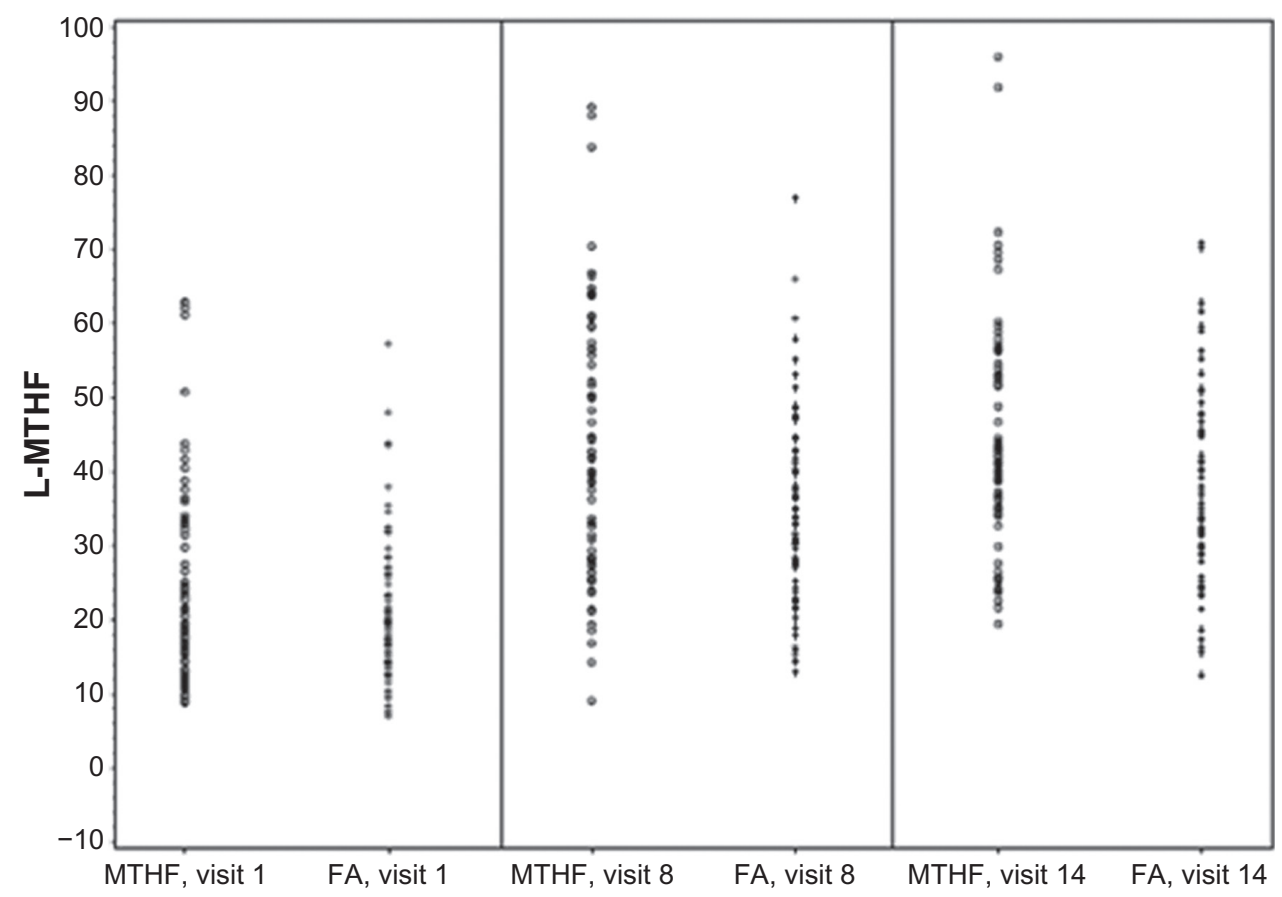

Figure 6 Distribution of individual L-MTHF plasma concentrations [nmol/L] at baseline (visit I), week I2 (visit 8), and week 24 (visit I4) after daily oral administration of EE-drospirenone-levomefolate calcium (MTHF) or EE-drospirenone + FA.

Note: Lower level of quantification for L-MTHF was between 3.325 and $3.680 \mathrm{nmol} / \mathrm{L}$.

Abbreviations: EE, ethinylestradiol; FA, folic acid; L-MTHF, L-5-methyl-tetrahydrofolate. 
Table 4 Mean metabolite concentrations in plasma (nmol/L)

\begin{tabular}{|c|c|c|c|c|}
\hline & \multicolumn{2}{|c|}{ EE-drsp-levomefolate calcium } & \multicolumn{2}{|l|}{ EE-drsp + folic acid } \\
\hline & THF & 5, I0-methenyl-THF & THF & 5, I0-methenyl-THF \\
\hline Baseline & $4.07 \pm 0.357(n=4)$ & $1.57 \pm 0.412(n=9)$ & $4.60 \pm 0.656(n=7)$ & $1.63 \pm 0.372(n=8)$ \\
\hline Week 12 & $4.98 \pm 1.85 \mid(n=13)$ & $1.76 \pm 0.603(n=7)$ & $5.28 \pm 1.806(n=14)$ & $1.45 \pm 0.282(n=7)$ \\
\hline Week 24 & $5.92 \pm 2.318(n=23)$ & $1.46 \pm 0.238(n=12)$ & $5.76 \pm 2.835(n=19)$ & $1.95 \pm 1.166(n=12)$ \\
\hline
\end{tabular}

Notes: Values are presented as the mean \pm standard deviation. Lower level of quantification = THF, between 3.447 and 3.539 nmol/L; 5, I0-methenyl-THF, between I.052 and $1.284 \mathrm{nmol} / \mathrm{L}$.

Abbreviations: drsp, drospirenone; EE, ethinylestradiol; THF, tetrahydrofolate.

\section{Pregnancies}

Two pregnancies occurred during the study. One volunteer became pregnant before the start of treatment (ie, during the screening period). This volunteer was not included in the study and delivered a healthy female at term. The second volunteer had a positive human chorionic gonadotropin test during the follow-up examination. A chemically-induced abortion was performed for social reasons. An ultrasound assessment in early pregnancy suggested that the volunteer became pregnant during intake of study medication in the elimination phase. A possible reason for this was an episode of diarrhea lasting 3 days approximately 2 weeks before the calculated date of conception (as determined by ultrasound).

\section{Safety}

The incidence of adverse events was similar between the EEdrospirenone-levomefolate calcium and EE-drospirenone + folic acid groups during the invasion phase and the elimination phase (Table 5). During the invasion phase, among the adverse events that were deemed possibly or probably related to the study medication, the most common were reproductive system and breast disorders (11 women [12.8\%] and eight women [9.3\%], respectively) and gastrointestinal disorders (six women [7.0\%] receiving EE-drospirenone-levomefolate calcium and none of the women receiving EE-drospirenone + folic acid). There were four serious adverse events (acoustic neuroma and impaired wound healing after surgery $[\mathrm{n}=1]$ in the EE-drospirenone-levomefolate calcium group;

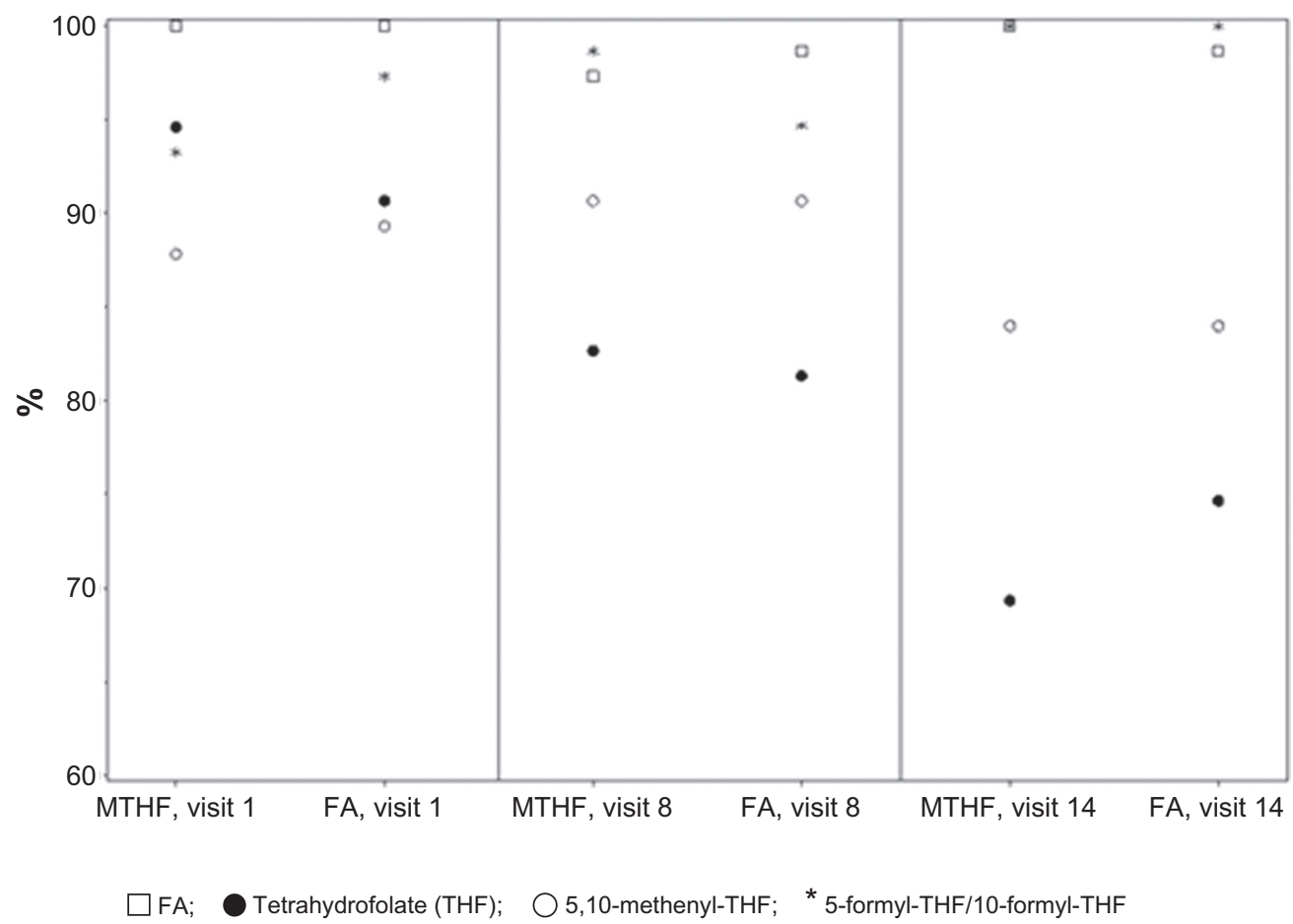

Figure 7 Percentages of metabolite values below lower limit of quantification at baseline (visit I), week I2 (visit 8), and week 24 (visit I4) after daily oral administration of EE-drospirenone-levomefolate calcium (MTHF) and EE-drospirenone + FA

Abbreviations: EE, ethinylestradiol; FA, folic acid; MTHF, 5-methyl-tetrahydrofolate. 
Table 5 Adverse events during the invasion phase, elimination phase, and the overall study (invasion + elimination phase)

\begin{tabular}{lll}
\hline & \multicolumn{2}{l}{ Treatment during invasion phase } \\
\cline { 2 - 3 } & $\begin{array}{l}\text { EE-drsp-levomefolate } \\
\text { calcium }\end{array}$ & $\begin{array}{l}\text { EE-drsp + } \\
\text { folic acid }\end{array}$ \\
\hline Invasion phase & & \\
AEs, \% & 96.5 & 95.3 \\
TEAEs, \% & 30.2 & 15.1 \\
SAEs, $n$ & 2 in I woman & 2 in 2 women \\
Elimination phase & & \\
AEs, \% & 87.5 & 91.5 \\
TEAEs, \% & 8.8 & 11.0 \\
SAEs, $n$ & 9 in 5 women & 3 in I woman \\
\hline
\end{tabular}

Abbreviations: $A E$, adverse event; drsp, drospirenone; $E E$, ethinylestradiol; $S A E$, serious $A E ;$ TEAE, treatment-emergent $A E$.

pyelonephritis $[\mathrm{n}=1]$ and ulcerative colitis $[\mathrm{n}=1]$ in the EE-drospirenone + folic acid group).

During the elimination phase, the most common treatment-related adverse events in the EE-drospirenone-levomefolate calcium group were "psychiatric disorders", occurring in $3.8 \%$ of women. In the EE-drospirenone + folic acid group, "reproductive system and breast disorders" were the most common treatment-related adverse events (7.3\%). The most common of these were "breast discomfort" and similar terms (eg, pain, swelling, tenderness), "metrorrhagia" and similar terms, and "dysmenorrhea". Nine serious adverse events occurred in five women in the EE-drospirenone-levomefolate calcium group (arthralgia, esophageal food impaction, appendicitis, abdominal pain lower, hemorrhagic ovarian cyst, abdominal pain, nausea, diarrhea, cholelithiasis). In the EE-drospirenone + folic acid group, three serious adverse events occurred in one woman (loss of consciousness, hyperventilation, alcohol poisoning). All serious adverse events were single occurrences and assessed as being unrelated to the study medication. General physical and gynecologic examinations as well as routine laboratory safety assessments did not give rise to any safety concerns.

\section{Discussion}

The invasion phase of this randomized, long-term study demonstrates that levomefolate calcium is a suitable alternative to folic acid for improving folate status when combined with an oral contraceptive in women of childbearing potential. This supports the concept that oral contraceptives represent a reasonable delivery vehicle for folate supplementation prior to pregnancy. Our results may be generalizable to other oral contraceptives, provided absence of interaction can be proven for the active components. The elimination phase of this study shows that 20 weeks after cessation of folate intake, an improved folate status is maintained in a large proportion of women.

Findings from the invasion phase, which are based on established surrogate parameters of folate status (plasma folate, $\mathrm{RBC}$ folate, and homocysteine), are consistent with two previous studies investigating the effects of levomefolate calcium and folic acid on folate levels in women of childbearing age. ${ }^{26,27}$ Data from a similarly designed study show that levomefolate calcium $0.451 \mathrm{mg}$ /day (equimolar to $0.416 \mathrm{mg}$ L-5-methyl-THF) and folic acid $0.400 \mathrm{mg} /$ day increased plasma folate concentrations to a comparable extent after 24 weeks. ${ }^{25}$ This randomized, controlled study involving 144 women of childbearing age found that administration of levomefolate calcium $0.451 \mathrm{mg} /$ day was more effective than folic acid $0.400 \mathrm{mg} /$ day at improving folate status. ${ }^{24} \mathrm{In}$ addition, as outlined above, the advantages of levomefolate calcium over folic acid are described in the literature. ${ }^{28}$

Results from the elimination phase of this study are consistent with earlier, exploratory studies examining the change in folate status following cessation of folate supplementation. ${ }^{32-34} \mathrm{Hao}$ et $\mathrm{al}^{33}$ examined the effect of 6 months of supplementation with oral folic acid (dose range $0.1-4 \mathrm{mg} /$ day) in 1108 Chinese women. At the end of the observation period of 3 months after cessation of supplementation, plasma and RBC folate levels remained higher than baseline, and homocysteine levels remained lower than baseline. ${ }^{33}$ In another study of 27 healthy women receiving $0.500 \mathrm{mg}$ folic acid/day for 8 weeks, plasma folate levels remained marginally above baseline and RBC folate levels clearly above baseline at the end of the observation period (12 weeks after cessation of supplementation). ${ }^{32}$

After 24 weeks of treatment with EE-drospirenonelevomefolate calcium or EE-drospirenone + folic acid during the invasion phase of this study, the geometric mean $\mathrm{AUC}_{(0-24 \text { weeks })}$ was generally comparable for plasma and $\mathrm{RBC}$ folate. Using bioequivalence criteria for comparisons, exposure was shown to be similar between the treatment groups for the plasma folate baseline-uncorrected and baseline-corrected values and for RBC folate baseline-uncorrected values.

Based on single-dose data with EE-drospirenonelevomefolate calcium ${ }^{35}$ and levomefolate calcium and folic acid, ${ }^{36}$ accumulation of plasma folate as observed in the current study would not be predicted after daily dosing because folate levels returned to baseline within a 24-hour dosing interval. Thus, the observed increase in plasma folate suggests a dynamic equilibrium between tissue and plasma, in which tissue folate stores build up over time with treatment. As tissue folate levels increase, the distribution of 
folate into plasma changes, which leads to increased plasma concentrations. After cessation of daily folate intake, plasma folate levels decline gradually and much more slowly than after single oral folate administration. ${ }^{36}$ This slow decrease is attributed in part to a re-equilibration between plasma and tissue, indicating a slow release of folate from tissue stores into the plasma. Although plasma folate concentrations during the elimination phase were mainly determined by distribution and elimination processes, RBC folate concentrations followed a different time course, indicating a different underlying mechanism. Indeed, in the present study, RBC folate levels were maintained at $\geq 906 \mathrm{nmol} / \mathrm{L}$ for a further 10 weeks (ie, week 34 of the study) in $50 \%$ of the subjects after discontinuation of EE-drospirenonelevomefolate calcium. When the proportion of patients with folate levels above baseline was examined, RBC folate levels were maintained above baseline for 20 weeks (ie, five cycles) after cessation of EE-drospirenone-levomefolate calcium in $89.3 \%$ of women.

Following oral administration, folic acid is absorbed in the proximal small intestine, metabolized during passage across the intestinal mucosa and into the liver and enters the peripheral circulation as L-5-methyl-THF. Orally administered L-5-methylTHF enters the systemic circulation as such. Therefore, any difference between folic acid and L-5-methyl-THF is expected to disappear as soon as folates enter the systemic circulation and enter the subsequent steps of metabolism. Based on this knowledge, oral administration of folic acid or L-5-methyl-THF would be expected to result in similar folate metabolite patterns in plasma. In the current study, all folate metabolites showed a qualitatively similar pattern between EE-drospirenonelevomefolate calcium and EE-drospirenone + folic acid at all time points throughout the invasion phase.

Elevated homocysteine concentrations have been shown to be a risk factor for low birth weight, premature delivery, pre-eclampsia, and neural tube defects. ${ }^{37}$ Previous studies have shown that levomefolate calcium is as effective as an equimolar dose of folic acid at lowering homocysteine concentrations. ${ }^{25,26}$ In agreement, the homocysteine-lowering effects of EE-drospirenone-levomefolate calcium and EEdrospirenone + folic acid were similar throughout the course of the invasion phase in this study.

In terms of timing of folate intake, this and several other studies have shown that it takes months to achieve steadystate plasma and RBC folate concentrations. The time point at which plasma folate levels reach a plateau seems to depend on baseline folate levels and sampling intervals, but the data suggest that a minimum of 8 weeks of treatment is needed in order to achieve steady-state concentrations. In a randomized controlled trial in a US population (in which baseline folate levels are higher due to mandatory food fortification), plasma folate levels reached a plateau after 8 weeks of treatment with EE $0.02 \mathrm{mg}$ - drospirenone $3 \mathrm{mg}$ - levomefolate calcium $0.451 \mathrm{mg} .{ }^{38}$ In other populations without mandatory food folate fortification, plasma folate levels reached a plateau after 12 weeks of treatment with folic acid or levomefolate calcium. ${ }^{24,33}$ Data on RBC folate levels indicate that a plateau is approached, but not completely reached, after 24 weeks of regular intake of folate supplements in populations without mandatory food folate fortification, ${ }^{24,33,39,40}$ a finding that is corroborated by the current study. Fetal neural tube defects occur early in pregnancy, within 28 days of conception, ${ }^{1}$ and the neural tube defect risk is directly linked to maternal folate status in the periconceptional period. ${ }^{3}$ Therefore, in order for a woman to maximize the reduction in neural tube defect risk, continuous folate intake needs to start well in advance of conception. Indeed, based on the results of long-term folate studies, Lamers et $\mathrm{al}^{24}$ recommended extending the period of periconceptional folic acid supplementation from 4 weeks to $\geq 12$ weeks to achieve maximal risk reduction. The results of the current study support this recommendation.

The addition of folate to an oral contraceptive represents a valuable means of boosting folate levels in women of reproductive age who unintentionally conceive during use of oral contraception or cease use of oral contraceptives in order to become pregnant. A prospective, post-marketing, noninterventional cohort study of new oral contraceptive users in clinical practice across seven European countries showed that of those who discontinue use of oral contraception to become pregnant, over one-fifth did so within one cycle after cessation of oral contraceptives and almost $50 \%$ after three cycles. ${ }^{41}$ The oral contraceptive plus folate combination represents a targeted approach to improve folate status specifically in women of childbearing potential.

During the elimination phase, a substantial proportion of women maintained folate levels that were higher than those at baseline, thus contributing to the reduction in neural tube defect risk. However, it is important that women who stop using a folate-containing oral contraceptive with the intention of becoming pregnant continue to receive adequate doses of folate. Indeed, the prescription of a folate-fortified oral contraceptive provides health care professionals with an opportunity to counsel women about the immediate need for adequate prenatal care following cessation of oral contraception.

All treatments were well tolerated, with no relevant differences observed between the groups. No serious adverse 
events were considered to be treatment-related and the tolerability profiles of EE-drospirenone-levomefolate calcium and EE-drospirenone + folic acid during the invasion phase were consistent with those of other oral contraceptives. ${ }^{42-44}$ Overall, these results confirm that addition of folate to an oral contraceptive does not give rise to additional safety concerns.

In summary, treatment with EE-drospirenone-levomefolate calcium for 24 weeks resulted in similar rates of increase and, following cessation of treatment, in similar rates of decrease in plasma and $\mathrm{RBC}$ folate levels compared with treatment using EE-drospirenone + folic acid. The effect on plasma homocysteine levels was also comparable between the two treatments. In a substantial proportion of women, folate levels remained above those at baseline for five oral contraceptive treatment cycles following cessation of 24 weeks of daily treatment with EE-drospirenone-levomefolate calcium. The risk of a neural tube defect-affected pregnancy would be reduced in women with improved folate status compared with their risk prior to the start of folate treatment.

\section{Acknowledgments}

Funding for this study was provided by Bayer HealthCare Pharmaceuticals, the manufacturer of EE $0.030 \mathrm{mg}$-drospirenone $3 \mathrm{mg}\left(\right.$ Yasmin $\left.^{\circledR}\right)$ and EE-drospirenone-levomefolate calcium. The authors are grateful to Klaus Pietrzik for his stimulating discussions concerning the study design. The authors would also like to thank Guenter Michl (bioanalytics), Susanne Reschke (clinical pharmacokinetics), and Vijay Chauhan (clinical statistics).

\section{Disclosure}

The authors would like to thank Claire Byrne of inScience Communications, Springer Healthcare, for medical writing support during preparation of the manuscript, which was funded by Bayer HealthCare Pharmaceuticals. KD, DT, FE, $\mathrm{BR}$, and HB are employees of Bayer HealthCare Pharmaceuticals. ML and MK are employees of Nuvisan $\mathrm{GmbH}$, Neu-Ulm, Germany.

\section{References}

1. Botto LD, Moore CA, Khoury MJ, Erickson JD. Neural tube defects. $N$ Engl J Med. 1999;341:1509-1519.

2. [No authors listed]. Prevention of neural tube defects: Results of the Medical Research Council Vitamin Study. MRC Vitamin Study Research Group. Lancet. 1991;338:131-137.

3. Daly LE, Kirke PN, Molloy A, Weir DG, Scott JM. Folate levels and neural tube defects. Implications for prevention. JAMA. 1995;274: 1698-1702.

4. Busby A, Abramsky L, Dolk H, et al. Preventing neural tube defects in Europe: a missed opportunity. Reprod Toxicol. 2005;20:393-402.
5. US Preventive Services Task Force. Folic acid for the prevention of neural tube defects: US Preventive Services Task Force recommendation statement. Ann Intern Med. 2009;150:626-631.

6. European Surveillance of Congenital Anomalies (EUROCAT). Folic acid special reports: survey of folic acid policy and practice in European countries, Dec 2007. Available from: http://www.eurocat-network.eu/ preventionandriskfactors/folicacid/folicacidspecialreports. Accessed January 24, 2013.

7. Smithells RW, Sheppard S, Schorah CJ, et al. Possible prevention of neural tube defects by periconceptional vitamin supplementation. Lancet. 1980;1:339-340.

8. Laurence KM, James N, Miller MH, Tennant GB, Campbell H. Doubleblind randomised controlled trial of folate treatment before conception to prevent recurrence of neural-tube defects. $\mathrm{Br}$ Med J (Clin Res Ed). 1981;282:1509-1511.

9. Mulinare J, Cordero JF, Erickson JD, Berry RJ. Periconceptional use of multivitamins and the occurrence of neural tube defects. JAMA. 1988;260:3141-3145.

10. Bower C, Stanley FJ. Dietary folate as a risk factor for neural-tube defects: evidence from a case-control study in western australia. Med J Aust. 1989;150:613-619.

11. Mitchell A. Case control study of neural tube defects and periconceptional vitamin use. Paediatr Perinat Epidemiol. 1989;3:216.

12. Vergel RG, Sanchez LR, Heredero BL, Rodriguez PL, Martinez AJ. Primary prevention of neural tube defects with folic acid supplementation: Cuban experience. Prenat Diagn. 1990;10:149-152.

13. Czeizel AE, Dudas I. Prevention of the first occurrence of neural-tube defects by periconceptional vitamin supplementation. $N$ Engl J Med. 1992;327:1832-1835.

14. Werler MM, Shapiro S, Mitchell AA. Periconceptional folic acid exposure and risk of occurrent neural tube defects. JAMA. 1993;269:1257-1261.

15. Shaw GM, Schaffer D, Velie EM, Morland K, Harris JA. Periconceptional vitamin use, dietary folate, and the occurrence of neural tube defects. Epidemiology. 1995;6:219-226.

16. Berry RJ, Li Z, Erickson JD, et al. Prevention of neural-tube defects with folic acid in china. China-US. Collaborative project for neural tube defect prevention. N Engl J Med. 1999;341:1485-1490.

17. De-Regil LM, Fernandez-Gaxiola AC, Dowswell T, Pena-Rosas JP. Effects and safety of periconceptional folate supplementation for preventing birth defects. Cochrane Database Syst Rev. 2010; 10: CD007950.

18. Mathews TJ, Honein MA, Erickson JD. Spina bifida and anencephaly prevalence - United States, 1991-2001. MMWR Recomm Rep. 2002;51:9-11.

19. Ray JG, Singh G, Burrows RF. Evidence for suboptimal use of periconceptional folic acid supplements globally. BJOG. 2004;111:399-408.

20. Tinker SC, Cogswell ME, Devine O, Berry RJ. Folic acid intake among US women aged 15-44 years, National Health and Nutrition Examination Survey, 2003-2006. Am J Prev Med. 2010;38:534-542.

21. Spina bifida and anencephaly before and after folic acid mandate United States, 1995-1996 and 1999-2000. MMWR Morb Mortal Wkly Rep. 2004;53:362-365.

22. US Food and Drug Administration. Summary minutes advisory committee for reproductive health drugs meeting, December 15, 2003. Available from: http://www.fda.gov/ohrms/dockets/ac/03/minutes/4002M1_Final. pdf. Accessed January 24, 2013.

23. Lassi ZS, Bhutta ZA. Clinical utility of folate-containing oral contraceptives. Int J Womens Health. 2012;4:185-190.

24. Lamers Y, Prinz-Langenohl R, Bramswig S, Pietrzik K. Red blood cell folate concentrations increase more after supplementation with [6s]-5methyltetrahydrofolate than with folic acid in women of childbearing age. Am J Clin Nutr. 2006;84:156-161.

25. Lamers Y, Prinz-Langenohl R, Moser R, Pietrzik K. Supplementation with [6s]-5-methyltetrahydrofolate or folic acid equally reduces plasma total homocysteine concentrations in healthy women. Am J Clin Nutr. 2004;79:473-478. 
26. Venn BJ, Green TJ, Moser R, Mann JI. Comparison of the effect of low-dose supplementation with 1-5-methyltetrahydrofolate or folic acid on plasma homocysteine: a randomized placebo-controlled study. Am J Clin Nutr. 2003;77:658-662.

27. Venn BJ, Green TJ, Moser R, McKenzie JE, Skeaff CM, Mann J. Increases in blood folate indices are similar in women of childbearing age supplemented with [6s]-5-methyltetrahydrofolate and folic acid. $J$ Nutr. 2002;132:3353-3355.

28. Pietrzik K, Bailey L, Shane B. Folic acid and 1-5-methyltetrahydrofolate: comparison of clinical pharmacokinetics and pharmacodynamics. Clin Pharmacokinet. 2010;49:535-548.

29. Yaz [prescribing information]. Berlin, Germany: Bayer HealthCare Pharmaceuticals Inc; 2012.

30. Pernet P, Lasnier E, Vaubourdolle M. Evaluation of the AxSym homocysteine assay and comparison with the IMX homocysteine assay. Clin Chem. 2000;46:1440-1441.

31. Smith DE, Kok RM, Teerlink T, Jakobs C, Smulders YM. Quantitative determination of erythrocyte folate vitamer distribution by liquid chromatography-tandem mass spectrometry. Clin Chem Lab Med. 2006;44:450-459.

32. Bakker DJ, de Jong-van den Berg LT, Fokkema MR. Controlled study on folate status following folic acid supplementation and discontinuation in women of child-bearing age. Ann Clin Biochem. 2009;46: 231-234.

33. Hao L, Yang QH, Li Z, et al. Folate status and homocysteine response to folic acid doses and withdrawal among young chinese women in a largescale randomized double-blind trial. Am J Clin Nutr. 2008;88:448-457.

34. Heseker H, Schmitt G. Effect of long-term supplementation of folate on folate status in plasma and erythrocytes. J Nutr Sci Vitaminol (Tokyo). 1987;33:163-168

35. Blode H, Klipping C, Richard F, Trummer D, Rohde B, Diefenbach K. Bioequivalence study of an oral contraceptive containing ethinylestradiol/ drospirenone/levomefolate calcium relative to $\mathrm{Yaz}^{\circledR}$ and to levomefolate calcium alone. Contraception. 2012;85:177-184.
36. Pentieva K, McNulty H, Reichert R, et al. The short-term bioavailabilities of [6s]-5-methyltetrahydrofolate and folic acid are equivalent in men. J Nutr. 2004;134:580-585.

37. Vollset SE, Refsum H, Irgens LM, et al. Plasma total homocysteine, pregnancy complications, and adverse pregnancy outcomes: The Hordaland Homocysteine Study. Am J Clin Nutr. 2000;71:962-968.

38. Bart SS, Marr J, Diefenbach K, Trummer D, Sampson-Landers C. Folate status and homocysteine levels during a 24-week oral administration of a folate-containing oral contraceptive: a randomized, double-blind, activecontrolled, parallel-group, us-based, multicenter study. Contraception. 2012;85:42-50.

39. Pietrzik K, Lamers Y, Bramswig S, Prinz-Langenohl R. Calculation of red blood cell folate steady state conditions and elimination kinetics after daily supplementation with various folate forms and doses in women of childbearing age. Am J Clin Nutr. 2007;86:1414-1419.

40. Hursthouse NA, Gray AR, Miller JC, Rose MC, Houghton LA. Folate status of reproductive age women and neural tube defect risk: the effect of long-term folic acid supplementation at doses of $140 \mu \mathrm{g}$ and $400 \mu \mathrm{g}$ per day. Nutrients. 2011;3:49-62.

41. Cronin M, Schellschmidt I, Dinger J. Rate of pregnancy after using drospirenone and other progestin-containing oral contraceptives. Obstet Gynecol. 2009;114:616-622.

42. Bachmann G, Sulak PJ, Sampson-Landers C, Benda N, Marr J. Efficacy and safety of a low-dose 24-day combined oral contraceptive containing 20 micrograms ethinylestradiol and $3 \mathrm{mg}$ drospirenone. Contraception. 2004;70:191-198.

43. Cronin M, Korner P. The safety profile of Yasmin is similar to other combined oral contraceptives. Clin Appl Thromb Hemost. 2008;14: 245-246.

44. Endrikat J, Muller U, Dusterberg B. A twelve-month comparative clinical investigation of two low-dose oral contraceptives containing 20 micrograms ethinylestradiol/75 micrograms gestodene and 30 micrograms ethinylestradiol/75 micrograms gestodene, with respect to efficacy, cycle control, and tolerance. Contraception. 1997;55:131-137.
International Journal of Women's Health

\section{Publish your work in this journal}

The International Journal of Women's Health is an international, peerreviewed open-access journal publishing original research, reports, editorials, reviews and commentaries on all aspects of women's healthcare including gynecology, obstetrics, and breast cancer. The manuscript management system is completely online and includes

\section{Dovepress}

a very quick and fair peer-review system, which is all easy to use. Visit http://www.dovepress.com/testimonials.php to read real quotes from published authors. 